\section{Pascal panretinal laser ablation and regression analysis in proliferative diabetic retinopathy: Manchester Pascal Study Report 4}

MMK Muqit',2, GR Marcellino3, DB Henson ${ }^{1,2}$, LB Young ${ }^{1}$, GS Turner ${ }^{1}$ and PE Stanga ${ }^{1,2}$

\begin{abstract}
Aims To quantify the 20-ms Pattern Scan Laser (Pascal) panretinal laser photocoagulation (PRP) ablation dosage required for regression of proliferative diabetic retinopathy (PDR), and to explore factors related to long-term regression.

Methods We retrospectively studied a cohort of patients who participated in a randomised clinical trial, the Manchester Pascal Study. In all, 36 eyes of 22 patients were investigated over a follow-up period of $\mathbf{1 8}$ months. Primary outcome measures included visual acuity (VA) and complete PDR regression. Secondary outcomes included laser burn dosimetry, calculation of retinal PRP ablation areas, and effect of patient-related factors on disease regression. A PDR subgroup analysis was undertaken to assess all factors related to PDR regression according to disease severity. Results There were no significant changes in logMAR VA for any group over time. In total, 10 eyes $(28 \%)$ regressed after a single PRP. Following top-up PRP treatment, regression rates varied according to severity: $75 \%$ for mild PDR $(n=6), 67 \%$ for moderate PDR $(n=14)$, and $43 \%$ in severe PDR $(n=3)$. To achieve complete disease regression, mild PDR required a mean of 2187 PRP burns and $264 \mathrm{~mm}^{2}$ ablation area, moderate PDR required 3998 PRP burns and area $456 \mathrm{~mm}^{2}$, and severe PDR needed 6924 PRP laser burns $\left(836 \mathrm{~mm}^{2}\right.$; $P<0.05$ ).

Conclusions Multiple 20-ms PRP treatments applied over time does not adversely affect visual outcomes, with favourable PDR regression rates and minimal laser burn
\end{abstract}

expansion over 18 months. The average laser dosimetry and retinal ablation areas to achieve complete regression increased significantly with worsening PDR.

Eye (2011) 25, 1447-1456; doi:10.1038/eye.2011.188; published online 5 August 2011

Keywords: Pascal panretinal photocoagulation; laser dosimetry; proliferative diabetic retinopathy

The Diabetic Retinopathy Study (DRS) established guidelines for primary panretinal laser photocoagulation (PRP) using argon and xenon laser in proliferative diabetic retinopathy (PDR). ${ }^{1,2}$ Longer-term reports including a DRS cohort study showed that the effectiveness of PRP treatment is dose related, and supplemental 'top-up' PRP is often required following primary treatment in order to achieve complete disease regression. ${ }^{3-5}$ In the United Kingdom, a Department of Health-funded National Diabetic Retinopathy Laser Treatment Audit evaluated PRP laser delivery in 1995, and reported that the clinical and visual outcomes is dependent on the surface area ablated with PRP laser. ${ }^{6,7}$

In current clinical practice, the parameters for PRP are evolving, with shorter pulse duration laser settings being used. ${ }^{8,9}$ However, there is no recent evidence that supports the effectiveness of 20-ms PRP treatments to completely regress PDR in the long term. We have previously undertaken a randomised clinical trial, the MAnchester Pattern Scan Laser (PAScal) Study (MAPASS). ${ }^{10}$ This trial consisted of a group of treatment-naive patients, matched against
${ }^{1}$ Retina Service, Manchester Royal Eye Hospital, Manchester, UK

${ }^{2}$ School of Medicine, University of Manchester, Manchester, UK

${ }^{3}$ OptiMedica Corporation, Santa Clara, CA, USA

Correspondence: PE Stanga, Retina Service, Manchester Royal Eye Hospital, Oxford Road, Manchester M13 9WL, UK Tel: +44 (0)161 276 1234; Fax: + 44 (0)161 2765555 E-mail: retinaspecialist@ btinternet.com

Received: 12 May 2011 Accepted: 27 June 2011 Published online: 5 August 2011 
Table 1 Study eye major inclusion and exclusion criteria for manchester pascal study
Inclusion criteria
- Older than 18 years of age
- Male or female patients with diabetes mellitus type I or type 2 who meet the WHO or ADA criteria for diabetes
- ETDRS visual acuity between 35 and 85 letters (Snellen equivalent of 6/60 or better)
- Newly diagnosed PDR
- Mean CRT of less than 300 microns as measured by OCT scans with absence of intra and/or subretinal fluid
- Adequate pupil dilatation and clear media to perform laser photocoagulation, digital photography and OCT scans
- Ability to perform accurate Humphrey visual field test
- If both eyes are eligible, then both eyes will be randomised as per protocol and treated independently
Exclusion criteria
- Recent (last 6 months) or ongoing poor glycaemic control. HbA1C greater than $10.0 \mathrm{mg} / \mathrm{dL}$
- Uncontrolled hypertension. Blood pressure greater or equal to $180 / 110 \mathrm{mmHg}$
- History of chronic renal failure or renal transplant for diabetic nephropathy
- Lens opacity/cataract that could influence vision and results
- Any previous surgical or laser treatment to the study eye or fellow eye
- Planned YAG peripheral iridotomy
- Previous laser photocoagulation or macular laser treatment to study eye or fellow eye
- History of DMO in study or fellow eye
- Any previous ocular condition that may be associated with a risk of macular oedema
- Active lid or adnexal infection
- Previous retinal treatment: laser, drug or surgery
- Planned intra-ocular surgery within one year

Abbreviations: WHO, World Health Organization; ADA, American Diabetic Association; ETDRS, Early Treatment Diabetic Retinopathy Study; PDR, proliferative diabetic retinopathy; CRT, retinal thickness within central subfield; OCT, optical coherence tomography; HbA1C, glycosylated hemoglobin; YAG, yttrium aluminum garnet; DMO, diabetic macular oedema.

eligibility criteria with baseline fluorescein angiography, and this would be an ideal cohort to examine over the long term. Following the MAPASS 12-week completion visit, all patients were managed as part of routine care in the retinal service at Manchester Royal Eye Hospital $(\mathrm{MREH})$. Patients with persistent, active PDR routinely underwent top-up Pascal 20-ms PRP treatment at MREH.

The main aims of this study included vision outcomes and quantification of the laser ablation dosage required by patients in the MAPASS trial cohort to achieve complete disease regression in the long term. The secondary aim was to explore predictor factors (patientrelated or disease-specific factors) for PDR-laser responses.

\section{Materials and methods}

The MAPASS was a prospective, randomised clinical trial that involved primary PRP for treatment-naive patients, with 3 months follow-up, undertaken at MREH between 23 June 2008 and 10 July 2009. The trial entry inclusion and exclusion criteria are outlined in Table 1. For this study, we retrospectively studied this cohort of patients over a longer follow-up period of 1-2 years. The study protocol received approval from Research Ethics Committee, and informed written consent was obtained from all participants. Data and safety monitoring were provided by an independent panel at both the University of Manchester and the Research Office at MREH. We certify that all applicable institutional and governmental regulations concerning the ethical use of human volunteers were followed during this research.

All study participants attended MREH for a final study visit. Demographic data capture included the following: age of patient; length of diabetes mellitus (months); type of diabetes (1 and 2); and a recent glycosylated haemoglobin (HbA1C) level within 3 months. We collected the following clinical data: visual acuity (VA) measured by a certified examiner using logMAR vision chart at $2 \mathrm{~m}$ by the ETDRS method; the total number of laser treatment visits; the total number of PRP burns applied to the treated eye; presence of vitreous haemorrhage; development of macular oedema; vitrectomy surgery intervention; cataract surgery; status of PDR disease activity and regression; driving status; and patient morbidity.

At the end of the MAPASS, each study eye had received a total of 1500 PRP treatment burns. At different time points during the follow-up period after MAPASS, patients underwent wide-field Optos fundus fluorescein angiography (WF-FFA; optomap fa, Dunfermline, Scotland) to assess the PDR disease activity as part of standard care at MREH. Wide-field Optos autofluorescence (WF-AF) imaging was undertaken in certain patients to assess panretinal laser coverage as part of standard care at MREH. 
All patients with active PDR underwent top-up PRP treatment using 20-ms pulse Pascal (Topcon Medical Laser Systems, Santa Clara, CA, USA) photocoagulation carried out by four experienced ophthalmologists using ETDRS guidelines. Threshold top-up PRP treatment was titrated to and designated by a mild grey-white burn (between grade $2+$ and $3+$ ) according to ETDRS guidelines at MREH. All treatments were performed in a single session under topical $0.4 \%$ oxybuprocaine hydrochloride, using 20-ms Pascal $5 \times 5,4 \times 4,3 \times 3$, or $2 \times 2$ multispot arrays. Burn distribution for top-up PRP treatment involved one burn width apart, greater than 2 disc diameters (DD) temporal to the fovea, no closer than one row within the arcades, and burn placement as close to the ora serrata as possible.

The following end points were evaluated in this study to explore relationships to the total laser required per treated eye. Primary end points included (1) final logMAR VA post laser and (2) complete inactivity/ regression of PDR disease, as determined by fluorescein angiogram and clinical assessment. Secondary end points included the following: (1) number of laser burns applied per $\mathrm{mm}^{2}$ retinal surface area; (2) effects of $\mathrm{HbA1C}$ over time (baseline $v$ s final level at follow-up); (3) ability of patients to pass DVLA driving visual field assessment following treatment; (4) wide-field autofluorescence imaging of PRP burns; (5) and the relationship of laser burn dosimetry and disease regression with patient factors, including the length of diabetes mellitus (months), type of diabetes (1 and 2), age of patient, and gender of patient.

\section{Assessment of disease regression}

In the original MAPASS, patients with any grade of treatment-naive PDR were recruited. ${ }^{10}$ The following grading scale was used at baseline: mild PDR (grade 1) - neovascularisation elsewhere (NVE) or at the disc (NVD) that was less than the standard Airlie House photograph 10A (SAH10A); moderate PDR (grade 2) -NVE greater than half a DD and/or NVD greater than SAH10A; and severe PDR (grade 3) - multiple NVE greater than half a DD, and/or forward NVD, and/or preretinal haemorrhage, and/or vitreous haemorrhage, and/or tractional retinal detachment. At baseline, the mean and median severity of PDR was moderate.

For this study, the following criteria were used to determine the disease activity. Complete PDR disease regression was defined by no leakage on WF-FFA, and/or disappearance of NV complexes on biomicroscopy with or without preretinal fibrosis. Active disease was shown using biomicroscopy and persistent leakage of NV complexes using WF-FFA, and this subgroup may develop vitreous haemorrhage and/or tractional retinal detachment.
Safety end points included all adverse events reported spontaneously by study participants, elicited by investigators, or observed by investigators. Adverse events were graded as mild, moderate, or severe, and were assessed as being either related to the laser intervention or unrelated to the laser treatment. The following were designated as expected adverse events in PDR patients undergoing laser therapy: diabetic macular oedema (DMO), vitreous haemorrhage, and tractional retinal detachment. The development of cataract is part of the ocular natural history for diabetes-related complications. We recorded all serious adverse events whether deemed related to the treatment or not, as per ethical and good clinical practice.

\section{Statistical analysis}

We performed statistical analyses using STATISTICA version 6 (2003; StatSoft Inc., Tulsa, OK, USA), and used the two-tailed $t$-test to explore changes in VA, laser burn dosage, and surface area ablation between each group. The null hypothesis was rejected for $P$-values $<0.05$.

\section{Results}

A total of 40 eyes of 24 patients were initially recruited into the MAPASS trial. In this follow-up study, 36 eyes of 22 patients were studied, with two patients (four eyes) excluded, as one died shortly after MAPASS completion and the other was lost to follow-up at MREH. The primary and secondary end points were analysed in three groups according to baseline grade of PDR (grades 1-3). Average follow-up was 15.3 months (range 6-24, group 1), 19.1 months (range 6-22, group 2), and 19.3 months (range 15-23) for group 3 . The study patient demographics are presented in Table 2.

\section{Visual acuity}

The mean baseline logMAR VAs were 0.04 (group 1, SD 0.04), 0.17 (group 2, SD 0.17), and 0.14 (group 3, SD 0.12). At final follow-up, there were no significant changes in $\log$ MAR VA for any group (group 1, SD 0.03; group 2, SD 0.16 ; and, group 3, SD 0.2 ). The effects of cataract surgery $(n=1)$ and vitrectomy $(n=1)$ did not significantly alter the VA outcomes. Overall, the application of top-up PRP laser did not adversely affect patient VA after an average 17.9 months follow-up for all groups.

\section{Proliferative disease regression}

Examples of disease regression post laser are shown in Figure 1. In group $1(n=8)$, the average age of patients 
Table 2 Patient characteristics for Follow-up Study

\begin{tabular}{|c|c|c|c|c|c|c|c|c|}
\hline $\begin{array}{l}\text { Study } \\
\text { eye }\end{array}$ & $\begin{array}{l}\text { Age } \\
\text { (years) }\end{array}$ & Sex & & & $\begin{array}{c}\text { Type of } \\
\text { diabetes }(1 / 2)\end{array}$ & $\begin{array}{l}\text { Length of } \\
\text { diabetes (years) }\end{array}$ & $\begin{array}{l}\text { PDR grade mild- } 1 \\
\text { moderate- } 2 \text { severe- } 3\end{array}$ & $\begin{array}{l}\text { Follow-up } \\
\quad \text { (mths) }\end{array}$ \\
\hline 1 & 29 & $\mathrm{M}$ & 8.5 & 7.8 & 1 & 29 & 2 & 24 \\
\hline 2 & 29 & $\mathrm{M}$ & 8.5 & 7.8 & 1 & 29 & 2 & 24 \\
\hline 3 & 59 & $\mathrm{M}$ & 7.5 & 6.4 & 2 & 3 & 2 & 24 \\
\hline 4 & 48 & $\mathrm{M}$ & 8 & 8.3 & 2 & 17 & 1 & 24 \\
\hline 5 & 42 & $\mathrm{M}$ & 7 & 6.7 & 1 & 30 & 2 & 24 \\
\hline 6 & 42 & $\mathrm{M}$ & 7 & 6.7 & 1 & 30 & 2 & 24 \\
\hline 7 & 49 & $\mathrm{M}$ & 6.3 & 7.1 & 2 & 8 & 3 & 18 \\
\hline 8 & 49 & $\mathrm{M}$ & 6.3 & 7.1 & 2 & 8 & 2 & 18 \\
\hline 9 & 37 & $\mathrm{M}$ & 10 & 7.8 & 1 & 18 & 3 & 23 \\
\hline 10 & 37 & $\mathrm{M}$ & 10 & 7.8 & 1 & 18 & 3 & 23 \\
\hline 11 & 35 & $\mathrm{~F}$ & 7.4 & 6.3 & 1 & 23 & 2 & 21 \\
\hline 12 & 53 & M & 7.2 & 9.1 & 2 & 20 & 2 & 22 \\
\hline 13 & 53 & $\mathrm{M}$ & 7.2 & 9.1 & 2 & 20 & 2 & 22 \\
\hline 14 & 34 & $\mathrm{~F}$ & 7 & 7.6 & 1 & 26 & 1 & 20 \\
\hline 15 & 34 & $\mathrm{~F}$ & 7 & 7.6 & 1 & 26 & 2 & 21 \\
\hline 16 & 34 & $\mathrm{M}$ & 9.2 & 8.1 & 1 & 26 & 2 & 6 \\
\hline 17 & 34 & M & 9.2 & 8.1 & 1 & 26 & 1 & 6 \\
\hline 18 & 59 & $\mathrm{~F}$ & 9.5 & 10.4 & 2 & 30 & 2 & 18 \\
\hline 19 & 52 & $\mathrm{M}$ & 5.5 & 6.2 & 1 & 10 & 2 & 22 \\
\hline 20 & 29 & $\mathrm{M}$ & 5.5 & 6.2 & 1 & 10 & 2 & 22 \\
\hline 21 & 53 & $\mathrm{~F}$ & 7 & 5.6 & 1 & 47 & 2 & 20 \\
\hline 22 & 51 & M & 9 & 9.2 & 1 & 22 & 3 & 20 \\
\hline 23 & 51 & $\mathrm{M}$ & 9 & 9.2 & 1 & 22 & 3 & 21 \\
\hline 24 & 30 & M & 8.1 & 6 & 1 & 10 & 2 & 17 \\
\hline 25 & 34 & $\mathrm{M}$ & 8 & 8 & 1 & 1 & 2 & 18 \\
\hline 26 & 44 & $\mathrm{~F}$ & 6 & 6.5 & 1 & 28 & 2 & 15 \\
\hline 27 & 44 & $\mathrm{~F}$ & 6 & 6.5 & 1 & 28 & 2 & 15 \\
\hline 28 & 49 & M & 8.8 & 7 & 1 & 30 & 1 & 15 \\
\hline 29 & 49 & M & 8.8 & 7 & 1 & 30 & 1 & 15 \\
\hline 30 & 48 & $\mathrm{~F}$ & 8.9 & 7.5 & 2 & 1 & 2 & 12 \\
\hline 31 & 48 & $\mathrm{~F}$ & 8.9 & 7.5 & 2 & 1 & 1 & 12 \\
\hline 32 & 51 & M & 9 & 8.2 & 2 & 5 & 3 & 15 \\
\hline 33 & 51 & M & 9 & 8.2 & 2 & 5 & 3 & 15 \\
\hline 34 & 35 & $\mathrm{M}$ & 9.2 & 7.2 & 1 & 30 & 1 & 15 \\
\hline 35 & 35 & $\mathrm{M}$ & 9.2 & 7.2 & 1 & 30 & 1 & 15 \\
\hline 36 & 70 & $\mathrm{M}$ & 6 & 6 & 2 & 11 & 2 & 12 \\
\hline
\end{tabular}

Abbreviations: HbA1C, glycosylated haemoglobin; PDR grade, proliferative diabetic retinopathy; Mild PDR, neovascularisation elsewhere (NVE) or at the disc (NVD) less than standard Airlie House photograph 10A (SAH10A); Moderate PDR, NVE greater than half disc diameter (DD) and/or NVD greater than SAH10A; Severe PDR, multiple NVE greater than half DD, and/or forward NVD, and/or preretinal haemorrhage, and/or vitreous haemorrhage.

was 42 years and the mean power of PRP used was $213 \mathrm{~mW}$ (range 104-350, SD 96). Six of eight eyes (75\%) showed complete regression after a mean interval of 5.8 months (range 3-10). In the two active cases, patients failed to comply with follow-up appointments at MREH since completion of MAPASS, and failed to attend for additional PRP laser on a number of occasions over a 14-month period. At the study visit, the grade of PDR was moderate with persistent NVE leakage on WF-FFA, and additional PRP laser was performed the same day as the study visit. There were no vitreous or retinal complications from PDR in this group.

For patients in group $2(n=14)$, the average age of patients was 42 years and the mean power of PRP used was $220 \mathrm{~mW}$ (range 116-482, SD 101). Complete PDR regression was present in $14 / 21$ eyes (67\%), and the mean time to regression was 11 months (range 3-19). Factors related to persistent active disease included worsening HbA1C control from 7.2 to $9.1 \mathrm{mg} / \mathrm{dl}$ over a 22-month period $(n=1)$; pregnancy-related worsening of PDR $(n=1)$; and, most commonly, failure to comply with follow-up appointments at MREH since completion of MAPASS resulted in worsening disease to PDR grade 3 NVD $(n=5)$. Four patients developed vitreous haemorrhage: a single patient underwent uncomplicated vitrectomy surgery; one case resolved spontaneously, and two eyes had persistent disease.

In the final group $3(n=7)$, the average age of patients was 47 years and the mean power of PRP used was $291 \mathrm{~mW}$ (range 140-398, SD 59). There was complete PDR 


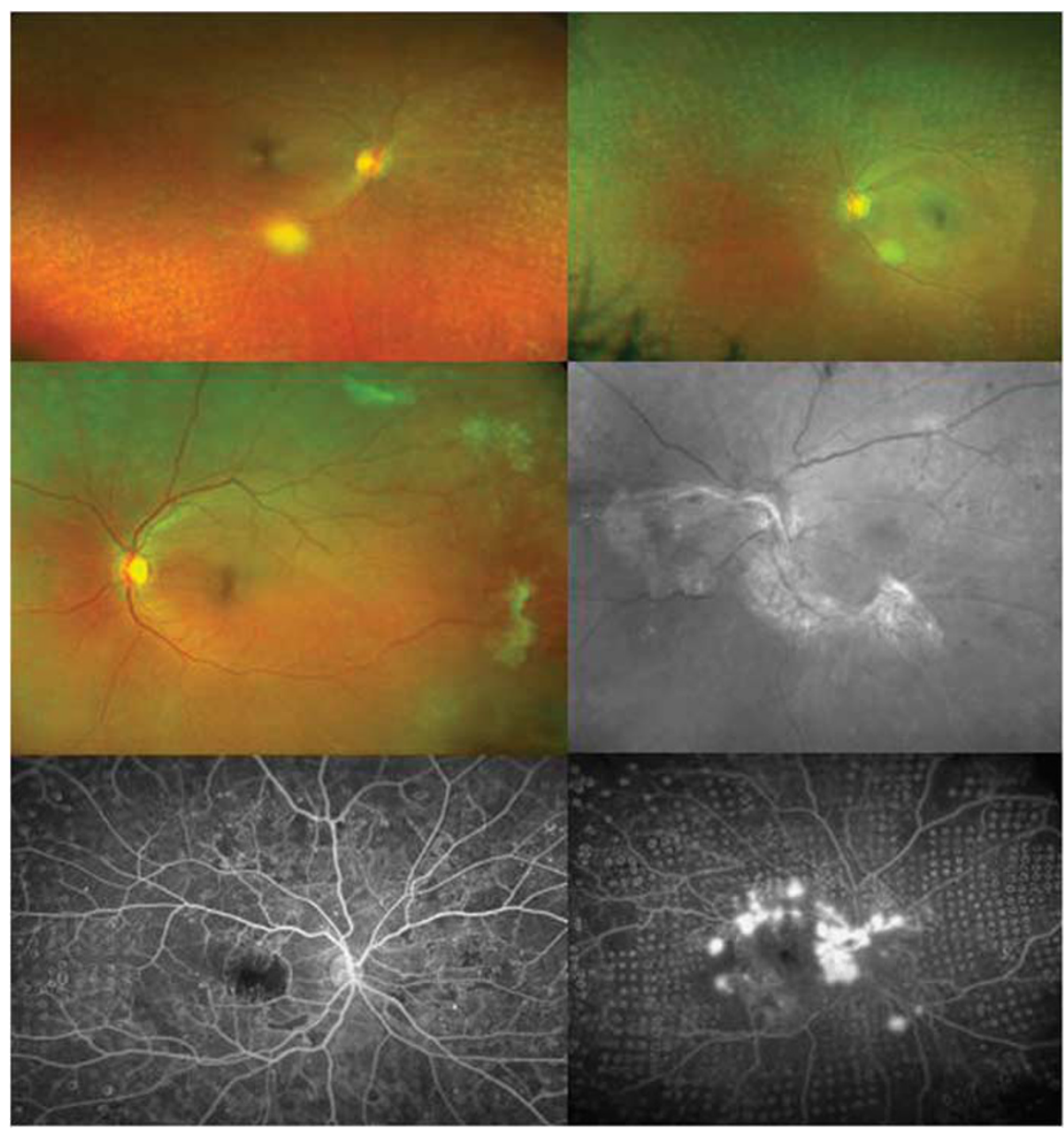

Figure 1 Wide-field Optos imaging of PDR treated with $20 \mathrm{~ms}$ PRP. Upper left: Colour image showing extensive PRP coverage and complete regression of moderate PDR, and fibrosis of NVE in the peripapillary area. Upper right: Colour image of extensive PRP coverage and complete regression of mild PDR, with involution of a nasal NVE complex. Middle left: Complete regression and fibrosis of multiple NVE complexes. Middle right: Red-free image showing a PRP laser non-responder with the presence of an extensive tractional retinal detachment arising from NVD. Lower left: Optos fluorescein angiogram showing complete regression of moderate PDR and absence of NVE leakage. Lower right: Optos fluorescein angiogram showing an example of severe PDR after a single session of PRP, with active leakage from multiple NVE and NVD.

regression in 3/7 eyes (43\%) over a mean period of 17 months. Suboptimal diabetic control (HbA1C >9\%) was the main factor for persistence of active PDR in one eye, persistent vitreous haemorrhage was detected in two eyes, and tractional retinal detachment occurred in the remaining cases (Figure 1 ).

\section{Laser burn dosimetry}

The total number of laser spots applied to each eye was calculated for each group (Figure 2). In group 1, the mean number of PRP burns used was 2187 (range 1500-3450, SD 785) delivered on average over two sessions (range 1-3 sessions), and this was significantly lower than group 2 (3998 burns delivered over four sessions; range 1500-8364; $P=0.012$ ). The highest number of burns was used in group 3, with a mean of 6924 burns (range 4097-9234; $P=0.012$ ) performed over six sessions (range 3-8).

The laser dosimetry required to produce complete PDR disease regression was analysed. For group 1, an average of 2416 burns $(n=6)$ was required, with 3902 burns for group $2(n=14)$, and 5446 laser PRP burns needed in group 3 (Figure 2 ).

\section{Laser burn ablation areas}

We calculated the PRP laser ablation area using methods described elsewhere. ${ }^{5,6}$ In this study, a Mainster 165 PRP lens (Ocular Instruments Inc., Bellevue, WA, USA) with a 1.96 magnification factor was used with a $200 \mu \mathrm{m}$ laser spot. The adjusted retinal laser spot size is $392 \mu \mathrm{m}$ 

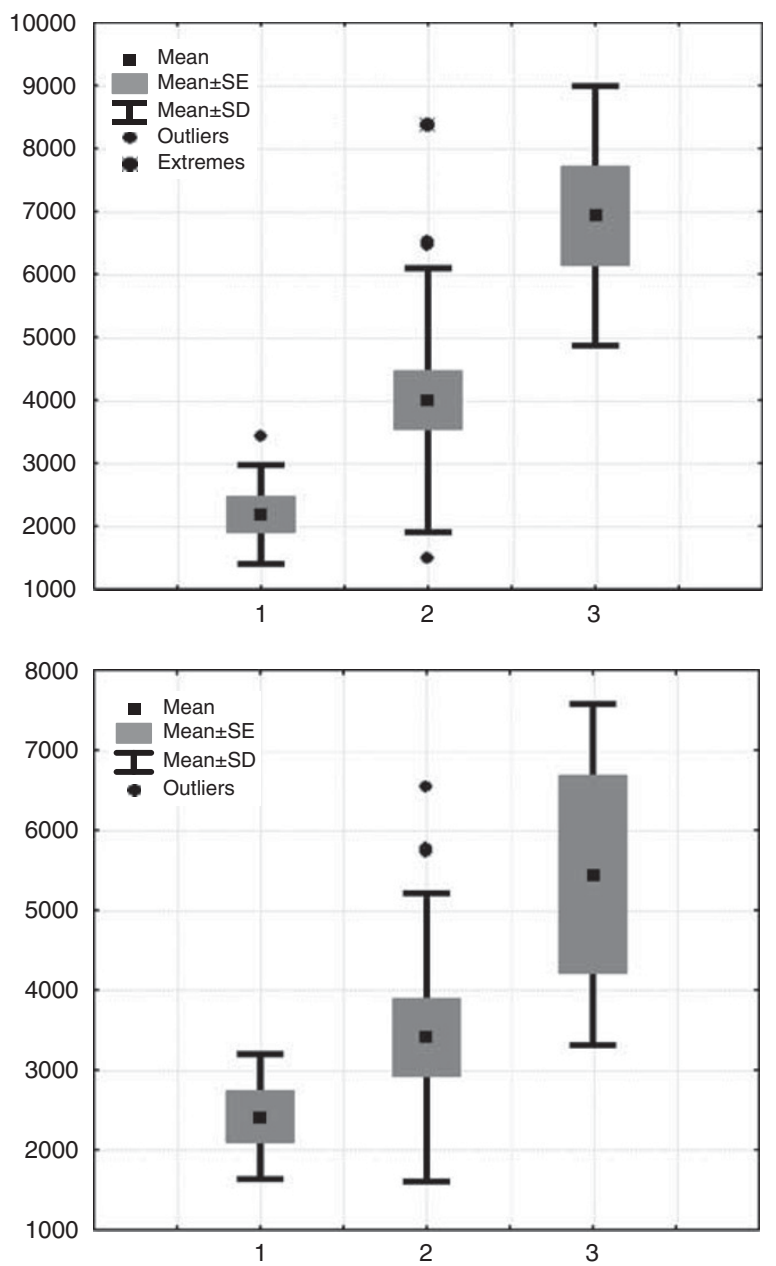

Figure 2 Laser burn dosimetry. Upper panel: Total number of laser burns applied according to PDR. $y$ axis: Number of laser burns. $x$ axis: Grade of PDR; mild -1 , moderate-2, and severe-3. Lower panel: Total number of laser burns required to produce complete regression of PDR disease. $y$ axis: Number of laser burns. $x$ axis: Grade of PDR; mild -1 , moderate -2 , and severe -3 .

$\left(1.96 \times 200 \mu \mathrm{m}^{2}\right)$ and the individual laser burn area calculated as $0.1206878 \mathrm{~mm}^{2}\left(\pi r^{2}\right)$. For example, a full 1500 -burn $(1500 \times 0.1206878)$ PRP used in the MAPASS trial produced a PRP laser ablation area of $181 \mathrm{~mm}^{2}$. We measured two parameters for PRP ablation area, namely the total retinal area ablated with PRP and also the total retinal ablation area treated to achieve complete PDR regression (Figure 3 ).

In group $1(n=8)$, patients received $181-416 \mathrm{~mm}^{2}$ laser ablation (SD 95, mean $264 \mathrm{~mm}^{2}$ ) treatment to achieve complete regression. Significantly larger PRP ablation areas were required in groups 2 and 3 . An average range of $181-1009 \mathrm{~mm}^{2}$ (SD 256, mean $456 \mathrm{~mm}^{2} ; P=0.036$ ) was treated in group $2(n=21)$, and $181-698 \mathrm{~mm}^{2}(n=14, \mathrm{SD}$ 264 , mean $471 \mathrm{~mm}^{2}$ ) was required to achieve regression. In the final group 3,523-1114 $\mathrm{mm}^{2}$ was treated in seven
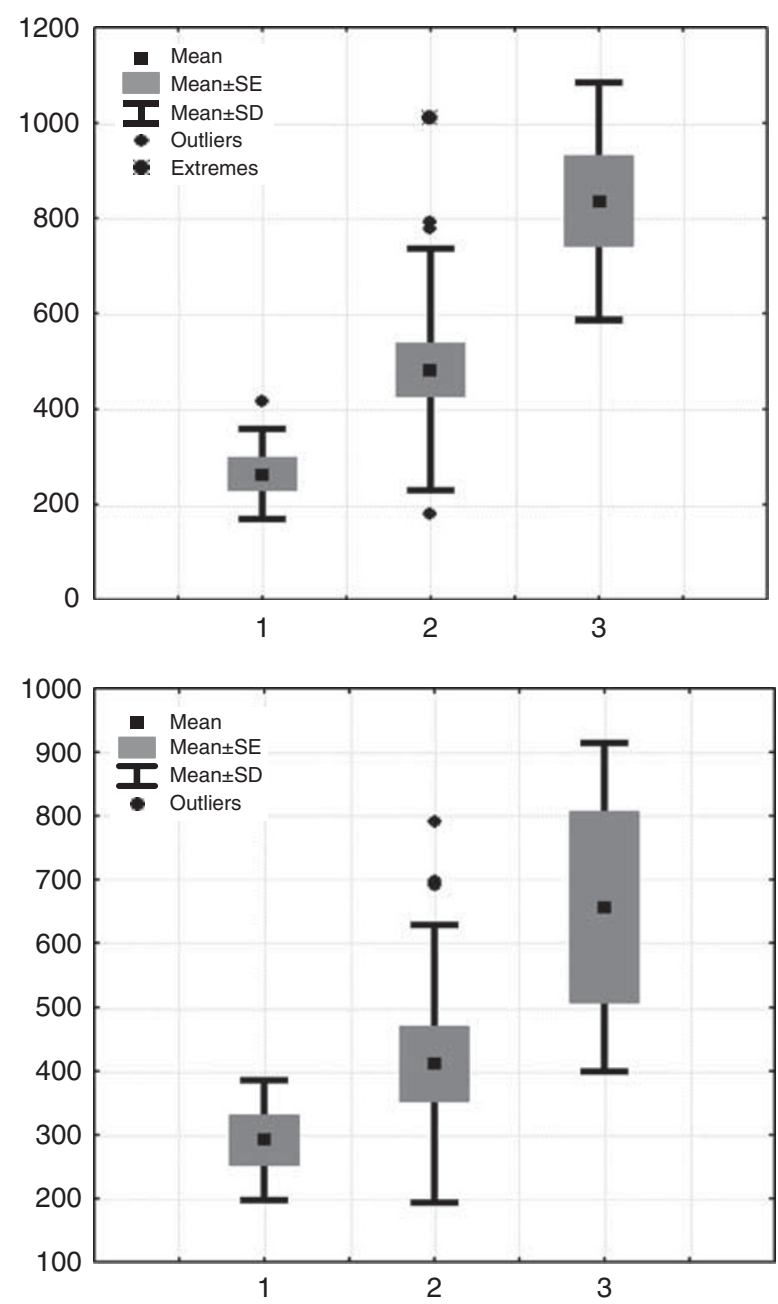

Figure 3 Upper panel: Total retinal ablation area treated with laser for PDR. $y$ axis: Retinal surface ablation area $\left(\mathrm{mm}^{2}\right) . x$ axis: Grade of PDR; mild - 1, moderate-2, and severe-3. Lower panel: Total retinal ablation area treated to produce complete regression of PDR disease. $y$ axis: Retinal surface ablation area $\left(\mathrm{mm}^{2}\right) . x$ axis: Grade of PDR; mild -1 , moderate -2 , and severe-3.

eyes (SD 249, mean $836 \mathrm{~mm}^{2} ; P=0.0091$ ) and $494-954 \mathrm{~mm}^{2}$ (mean $657 \mathrm{~mm}^{2}$, SD 258) was required to achieve regression.

\section{Wide-field autofluorescence imaging of laser burns}

The Optos colour images showed well-localised and partially pigmented PRP laser lesions that were barely visible on biomicroscopy (Figure 4). Application of topup 20-ms PRP treatment was often guided by WF-AF imaging, as the laser lesions were better visualised using this technique. This showed minimal changes in autofluorescence signals 6 months to 2 years post PRP. In some patients, there were areas lacking autofluorescence, which corresponded to laser burns, but more commonly, 


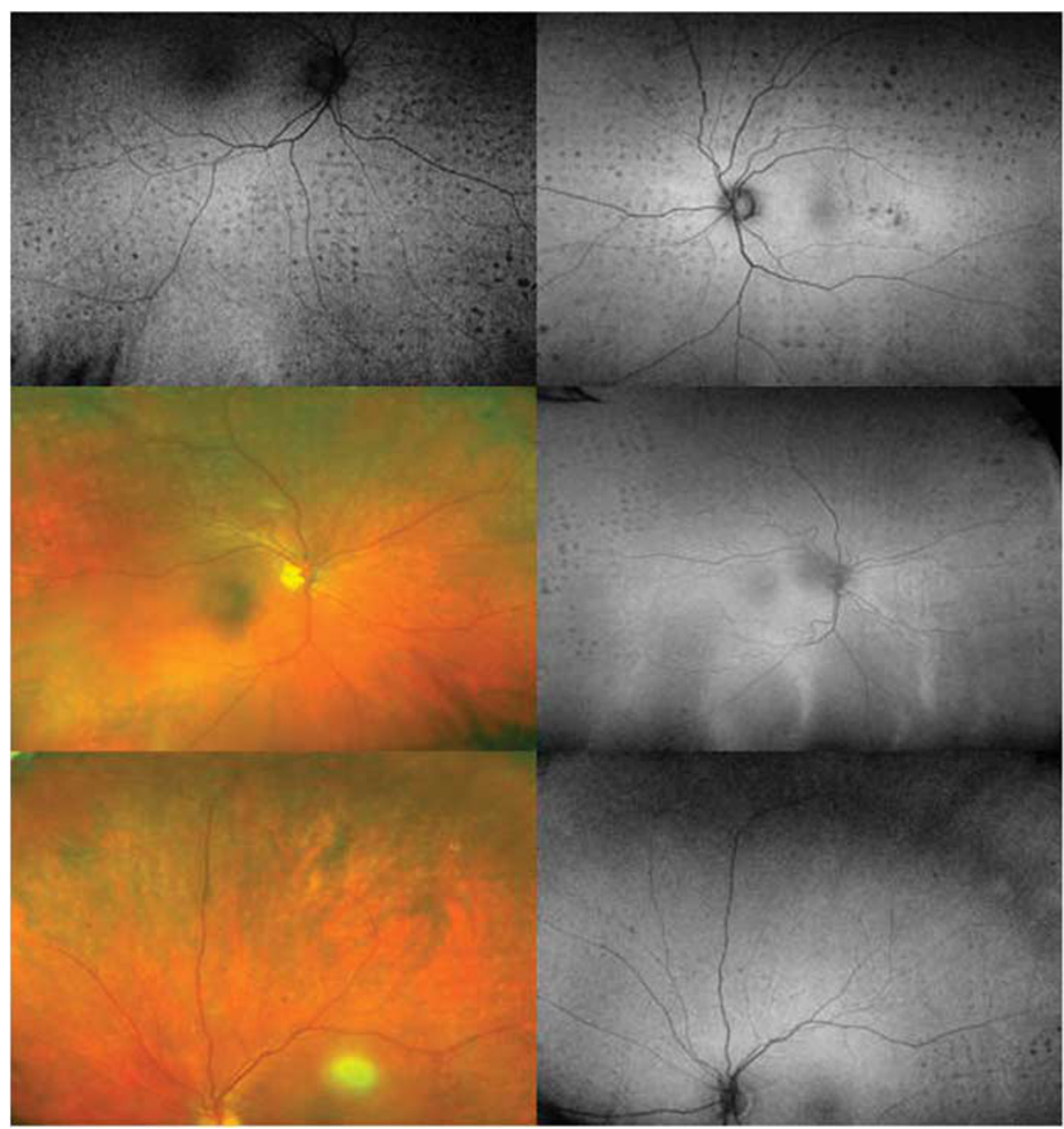

Figure 4 Optos colour and autofluorescence images of treated proliferative disease. Upper right: At 22 months, the areas lacking autofluorescence correspond to laser burns. Upper left: At 12 months, the Pascal $5 \times 5$ multispot arrays contain laser lesions lacking autofluorescence of mixed spatial density, and there is no significant laser burn expansion. Middle left: Colour image showing partially visible laser burns at 17 months post laser. Middle right: Peripheral retinal zones show small areas lacking autofluorescence that map the panretinal laser coverage on the colour image (middle left). Lower left: At 22 months following primary panretinal photocoagulation, the colour image shows barely visible panretinal laser burns. Lower right: Autofluorescence image of the same patient (lower left), and the laser lesions show minimal changes in autofluorescence imaging and no signs of laser burn expansion at 22 months post laser.

we observed that the 20-ms PRP laser lesions were not visible on WF-AF imaging and this is likely secondary to healing responses reported previously (Figure 4).

\section{Patient factors}

The secondary end points are shown in Table 1 . The average length of diabetes mellitus was 24 years (group 1), 20 years (group 2), and 14 years (group 3 ). There were no differences between groups for this factor, and no associations detected between either PRP laser dosage or VA changes and a patient's length of diabetes mellitus. Similarly, we found no associations between the PDR grade and disease response to PRP laser, and patient gender and type of diabetes mellitus in this study.
There was a significant change in $\mathrm{HbA1C}$ from baseline to final follow-up for group 1 patients $(-0.96 \mathrm{mg} / \mathrm{dl} ; P=0.0353)$. In the other groups, no significant alterations in mean $\mathrm{HbA} 1 \mathrm{C}$ were found between baseline and the final visit (group 2, $-0.08 \mathrm{mg} / \mathrm{dl}$, $P=0.736$; group $3,-0.69 \mathrm{mg} / \mathrm{dl}, P=0.176$ ).

Patients who had undergone bilateral PRP treatment in the study $(n=14)$ were questioned about the status of fitness to drive according to DVLA (UK) requirements. All patients had undergone independent VA and binocular VF testing within 6 months of the final study visit. At this visit, 13/14 patients reported passing the DVLA driving standards, and a single patient had failed because of suboptimal VA level, despite a satisfactory binocular VF test. The average number of PRP burns 
applied in this subgroup was 4476 (range 1500-9234), equivalent to a mean PRP ablation area of $518 \mathrm{~mm}^{2}$.

\section{Complications}

There were no reported unexpected adverse or serious adverse events for any patient in this study. In the short and long term, there were no signs of intraretinal/ subretinal haemorrhage or blood vessel damage from 20-ms PRP burns, and no indirect laser-related ocular complications reported.

All eyes with vitreous haemorrhage $(n=7)$ and tractional retinal detachment $(n=1)$ were associated with elevated/forward NVD greater than SAH10A. An uncomplicated, complete posterior vitreous detachment developed in three eyes, and the PDR was inactive at final follow-up. Over 6 months after completion of MAPASS, three eyes developed DMO. These events were unrelated to top-up PRP treatments, with one patient developing pregnancy-related DMO.

\section{Discussion}

We report the long-term visual and clinical outcomes for patients with PDR who underwent 20-ms PRP treatment. Visual outcomes were favourable after top-up 20-ms PRP treatment for all grades of PDR in this study. Following a primary PRP treatment of 1500 treatment burns, an additional 1000- to 2000-burn PRP was required in a single session to completely regress mild PDR. The laser burn density and retinal ablation areas increase significantly for moderate and severe PDR. Overall regression rates for PDR showed between 67 and $75 \%$ for mild/moderate PDR and $43 \%$ in grade 3 disease. In eyes non-responsive to repeated PRP, elevated/forward NVD greater than SAH10A was present with vitreous haemorrhage and/or tractional retinal detachment. ${ }^{11}$

The authors previously reported that 20-ms laser burns decrease in size over time, and the uncertainty remains in clinical practice as to whether a higher number of 20-ms PRP burns would be required to adequately treat PDR compared with conventional PRP guidelines. ${ }^{12,13}$ In this study, we have shown that a significant increment in 20-ms laser burn density and retinal ablation area are often required to treat and completely regress worsening grades of PDR. Standard 20-ms Pascal PRP regimens at MREH involved between 2000 and 7000 laser burns delivered over 2-7 hospital visits to treat PDR, and this laser treatment density falls within the DRS recommendations, stating that a higher number of laser burns are required to prevent visual loss in higher-risk PDR. ${ }^{14}$

The ETDRS recommended multisession $500 \mu \mathrm{m}$ PRP laser extending into far-peripheral zones in high-risk eyes. ${ }^{15}$ The DRS study recommended a minimum laser ablation area of $236 \mathrm{~mm}^{2}$ (range $157-314 \mathrm{~mm}^{2}$ ) for standard PRP, and the ETDRS suggested a minimum area of $236 \mathrm{~mm}^{2}$ for PRP treatment. In the United Kingdom, single-session PRP involved a median treatment area of $98.2 \mathrm{~mm}^{2}$ (range $6.7-682.5 \mathrm{~mm}^{2}$ ). ${ }^{6}$ At the time of the UK study in 1995, there appeared to be a trend to initially undertreat eyes compared with the DRS and ETDRS recommendations; however, subsequent PRP was often needed in clinical practice. . $^{3,4} 8$

The use of 1500 20-ms PRP burns in a single session was shown to be a safe regimen in the MAPASS trial. ${ }^{10}$ However, for long-term PDR regression, we found that $72 \%$ of eyes required top-up PRP treatment, and the laser burn treatment density and final treatment areas varied according to the risk profile of the PDR. ${ }^{16}$ Using 20-ms PRP treatment, the retinal ablation areas needed to produce complete disease regression ranged from 292 to $657 \mathrm{~mm}^{2}$. Importantly, the final visual outcomes for all grades of PDR were favourable in this study, with VA remaining within one line of pretreatment values. We identified disease-specific and patient-related factors for inadequate disease regression, including aggressive grade 3 PDR with NVD, suboptimal long-term HbA1C control, non-compliance with hospital follow-up, and pregnancy in one case.

The application of 20-ms PRP burns reduces the risk of overlapping laser scars, as the laser burns show healing responses over time. ${ }^{13}$ Calculation of the total retinal area has produced estimates between 1100 and $1368 \mathrm{~mm}^{2} .{ }^{17-19}$ Barr ${ }^{17}$ reported that a maximal number of 5500 laser burns could be applied to the retinal surface using a $500 \mu \mathrm{m}$ laser spot size. In 1995, Reddy and co-workers ${ }^{5}$ quantified the ablation areas using $500 \mu \mathrm{m}$ conventional argon PRP laser. In this large study, they reported that 2600-6500 laser burns, with a retinal coverage of $510-1280 \mathrm{~mm}^{2}$, is required to treat PDR with PRP dosage proportional to the number of retinopathy risk factors. Our results for 20-ms PRP treatment show results similar to this study; however, Reddy and co-workers used krypton red and/or cryotherapy as supplemental treatment modalities. In a study of eyes with NVD only, Aylward and co-workers ${ }^{20}$ reported $64 \%$ complete regression using conventional (200-500 $\mu \mathrm{m})$ 100-ms PRP treatment; however, a mean of 7225 laser shots were required (range 5279-11 513 burns) and this resulted in confluent laser scarring. The application of confluent PRP is outwith the current Royal College of Ophthalmologists recommended laser treatment guidelines. ${ }^{21}$

Despite an absence of previous published reports for long-term outcomes following Pascal $20 \mathrm{~ms}$ PRP in PDR, a study by Al-Hussainy and co-workers ${ }^{22}$ in Birmingham (UK) investigated $20 \mathrm{~ms}$ PRP using a conventional frequency-doubled neodymium-doped yttrium 
aluminium garnet system. This paper mainly highlights reduced pain responses, and we have analysed the laser dosage and follow-up data described in the article. In their study, an average of 3031 laser burns (range 1300-6600) was required to achieve complete PDR disease regression over a mean follow-up period of 20 months (range 6-45). Our study demonstrates that an average of 3921 PRP burns (range 2416-5446) are required to produce complete PDR regression of any grade using 20-ms pulse duration. We have shown with Optos imaging for the first time that multiple 20-ms PRP treatments producing an ablation area of $\sim 518 \mathrm{~mm}^{2}$ does not lead to extensive chorioretinal scarring on wide-field autofluorescence imaging. Laser burns at $20 \mathrm{~ms}$ have reduced fluence at all clinical grades of burn compared with 100 and $200 \mathrm{~ms}$, and $20 \mathrm{~ms}$ lesions undergo healing responses. This scar minimisation and retinal tissue preservation perhaps may explain why no patients undergoing bilateral top-up PRP treatment in this study failed UK DVLA VF driving standards.

There are a number of weaknesses and certain strengths to this study. The initial RCT was prospective, with patients matched to entry criteria, good patient reattendance for follow-up, with complete data retrieval for all patients studied. After the MAPASS trial, all patients returned to routine care at MREH outwith any study protocols or restrictions. During the post-MAPASS follow-up, PDR outcomes, WF-FFA reporting, and decisions to re-treat with PRP were undertaken by clinicians masked to these study objectives, thereby reducing the chances of any bias in reporting clinical outcomes by the current study investigators. The MREH laser guidelines involved a standardised Pascal 20-ms top-up PRP technique undertaken by all laser operators. We reassessed the patient diabetic control through posttreatment $\mathrm{HbA} 1 \mathrm{C}$ measurements, and this enabled us to evaluate this potential confounding factor. Limitations included the small study sample size, and so statistical power was too small to detect any differences between the relationships of patient-related factors and laser dosimetry/regression outcomes. Blood pressure was not recorded for this study; however, there were no significant effects in the MAPASS trial, and there were no documented changes in antihypertensive medication during follow-up for any patient. Estermann binocular VFs were not assessed at final follow-up, but all patients with bilateral PRP self-reported that they had been independently assessed in the community via the UK DVLA pathway.

Our study reports favourable visual and clinical effects for primary and top-up 20-ms PRP therapy using the Pascal system over the long term. The retinal ablation areas in eyes that completely regressed were greater than that recommended by the DRS. Wide-field autofluorescence imaging demonstrates minimisation of scar formation after repeated PRP treatments. Despite complete retinal laser coverage in PDR, high-risk NVD often has a poor prognostic outcome and can lead to patients becoming non-responders to additional PRP.

\section{Summary}

What was known before

- Favourable short-term visual and clinical outcomes are reported in the literature following 20-millisecond panretinal laser photocoagulation for proliferative diabetic retinopathy. There are no published studies that have investigated visual acuity outcomes, laser burn dosimetry, and retinal laser ablation required to successfully treat the condition over the long-term.

What this study adds

- Top-up Pascal 20-millisecond panretinal laser produced complete regression of mild to moderate disease in 67$75 \%$ of eyes studied. The laser burn density ranges from 2000 to 7000 burns and is significantly higher for moderate to severe disease, without any significant impact on visual acuity at 1-2 years follow-up. Despite an average retinal laser ablation area of $836 \mathrm{~mm}^{2}$ in severe proliferative retinopathy, $57 \%$ of eyes were non-responders to treatment. Elevated disc neovascularisation was a poor prognostic factor in this study for high-risk eyes.

\section{Conflict of interest}

GR Marcellino is an employee of Optimedica Corporation. PE Stanga has received financial support from Optimedica Corporation.

\section{Acknowledgements}

This research has been supported by the Manchester Academic Health Sciences Centre (MAHSC) and NIHR Manchester Biomedical Research Centre. This research was funded by Optimedica Corporation, Santa Clara, CA, USA.

\section{References}

1 Diabetic Retinopathy Study Group. Photocoagulation treatment of proliferative diabetic retinopathy. The second report of Diabetic Retinopathy Study findings. Ophthalmology 1978; 85: 82-106.

2 Diabetic Retinopathy Study Research Group. Photocoagulation treatment of proliferative diabetic retinopathy: clinical application of Diabetic Retinopathy Study findings, DRS report number 8. Ophthalmology 1981; 88: 533-600. 
3 Little HL. Treatment of proliferative diabetic retinopathy. Long-term results of argon laser photocoagulation. Ophthalmology 1985; 92(2): 279-283.

4 Kaiser RS, Maguire MG, Grunwald JE, Lieb D, Jani B, Brucker AJ et al. One-year outcomes of panretinal photocoagulation in proliferative diabetic retinopathy. Am J Ophthalmol 2000; 129(2): 178-185.

5 Reddy VM, Zamora RL, Olk RJ. Quantification of retinal ablation in proliferative diabetic retinopathy. $A m \mathrm{~J}$ Ophthalmol 1995; 119(6): 760-766.

6 Bailey CC, Sparrow JM, Grey RHB, Cheng H. The national diabetic retinopathy laser treatment audit. II. Proliferative retinopathy. Eye (Lond) 1998; 12(1): 77-84.

7 Bailey CC, Sparrow JM, Grey RHB, Cheng H. The national diabetic retinopathy laser treatment audit. III. Clinical outcomes. Eye (Lond) 1999; 13: 151-159.

8 Diabetic Retinopathy Clinical Research Network. Brucker AJ, Qin H, Elman MJ, Glassman AR, Gross JG, Kollman C et al. Observational study of the development of diabetic macular edema following panretinal (scatter) photocoagulation given in 1 or 4 sittings. Arch Ophthalmol 2009; 127(2): 132-140.

9 Muqit MMK, Sanghvi C, McLauchlan R, Delgado C, Young LB, Charles SJ et al. Study of clinical applications and safety for Pascal ((R)) laser photocoagulation in retinal vascular disorders. Acta Ophthalmol 2010; e-pub ahead of print 16 February 2010; doi:10.1111/j.1755-3768.2009.01854.x.

10 Muqit MMK, Marcellino GR, Henson DB, Young LB, Patton $\mathrm{N}$, Charles SJ et al. Single-session vs multiple-session pattern scanning laser panretinal photocoagulation in proliferative diabetic retinopathy. Arch Ophthalmol 2010; 128(5): 525-533.

11 Four risk factors for severe visual loss in diabetic retinopathy. The third report from the Diabetic Retinopathy Study. The Diabetic Retinopathy Study Research Group. Arch Ophthalmol 1979; 97: 654-655.

12 Muqit MM, Gray JC, Marcellino GR, Henson DB, Young LB, Patton $\mathrm{N}$ et al. In vivo laser-tissue interactions and healing responses from 20- vs 100-millisecond pulse Pascal photocoagulation burns. Arch Ophthalmol 2010; 128(4): 448-455.

13 Muqit MM, Denniss J, Nourrit V, Marcellino GR, Henson DB, Schiessl I et al. Spatial and spectral imaging of retinal laser photocoagulation burns. Invest Ophthalmol Vis Sci 2011; 52(2): 994-1002.

14 Kaufman SC, Ferris III FL, Seigel DG, Davis MD, DeMets DL. Factors associated with visual outcome after photocoagulation for diabetic retinopathy. Diabetic Retinopathy Study Report No. 13. Invest Ophthalmol Vis Sci 1989; 30(1): 23-28.

15 Diabetic Retinopathy Study Research Group. Photocoagulation treatment of proliferative diabetic retinopathy. Clinical application of Diabetic Retinopathy Study (DRS) findings, DRS report number 8. Ophthalmology 1981; 88(7): 583-600.

16 Doft BH, Metz DJ, Kelsey SF. Augmentation laser for proliferative diabetic retinopathy that fails to respond to initial panretinal photocoagulation. Ophthalmology 1992; 99(11): 1728-1734; discussion 1734-1735.

17 Barr CC. Estimation of the maximum number of argon laser burns possible in panretinal photocoagulation. Am J Ophthalmol 1984; 97(6): 697-703.

18 Taylor E, Jennings A. Calculation of total retinal area. Br J Ophthalmol 1971; 55(4): 262-265.

19 Drasdo N, Fowler CW. Non-linear projection of the retinal image in a wide-angle schematic eye. Br J Ophthalmol 1974; 58(8): 709-714.

20 Aylward GW, Pearson RV, Jagger JD, Hamilton AM. Extensive argon laser photocoagulation in the treatment of proliferative diabetic retinopathy. Br J Ophthalmol 1989; 73(3): 197-201.

21 Royal College of Ophthalmologists. Guidelines for Diabetic Retinopathy. RCOphth: London, 2005, pp 54-61.

22 Al-Hussainy S, Dodson PM, Gibson JM. Pain response and follow-up of patients undergoing panretinal laser photocoagulation with reduced exposure times. Eye (Lond) 2008; 22(1): 96-99. 